\title{
REGGE CALCULUS IN THE CANONICAL FORM
}

\section{Vladimir Khatsymovsky円}

$(3+1)$ (continuous time) Regge calculus is reduced to Hamiltonian form. The constraints are classified, classical and quantum consequences are discussed. As basic variables connection matrices and antisymmetric area tensors are used supplemented with appropriate bilinear constraints. In these variables the action can be made quasipolinomial with arcsin as the only deviation from polinomiality. In comparison with analogous formalism in the continuum theory classification of constraints changes: some of them disappear, the part of I class constraints including Hamiltonian one become II class (and vice versa, some new constraints arise and some II class constraints become I class). As a result, the number of the degrees of freedom coincides with the number of links in 3-dimensional leaf of foliation. Moreover, in empty space classical dynamics is trivial: the scale of timelike links become zero and spacelike links are constant.

\footnotetext{
${ }^{1}$ Budker Institute of Nuclear Physics, Novosibirsk 630090, Russia
} 


\section{INTRODUCTION.}

Regge calculus [1] attracts much attention in connection with possibility to construct quantum gravity theory free of ultraviolet divergences. Such the possibility is usually connected with discrete nature of the set of field variables. The latter are link lengths of flat tetrahedrons forming piecewiseflat Regge manifold. To introduce canonical quantisation we need continuous time Hamiltonian formalism. It was studied in a number of works [2]-[7]. My strategy is that of refs. [3, 8], in which required formalism is the limit of 4-dimensional Regge calculus while distances between successive spacelike leaves tend to zero. The main problem is an adequate choice of variables allowing one to describe in the continuous time limit all the degrees of freedom of an arbitrary Regge manifold and to pass to Hamiltonian formalism in the simplest way.In refs. [11] tetrad-connection variables were used first considered by Bander in ref. [12]. In [11 formulation of Regge calculus was suggested analogous to Einstein-Cartan formalism in the continuum general relativity $(\mathrm{GR})$ and, by passing to the continuous time limit, Lagrangian was found, although not for quite general Regge manifold. Using these results some trivial lowdimensional models were considered in refs. [13] illustrating possible versions of arising finite quantum theory.

In the given paper Einstein action for arbitrary Regge manifold is considered in the continuous time limit and reduced to the canonical form.

\section{DESCRIPTION OF THE SYSTEM.}

Our main object is Regge manifold or simplicial complex 15. The vertices or null-dimensional simplices $\sigma^{0}$ will be denoted by capital letters $A, B, C, \ldots ; n$-simplex $\sigma^{n}$ (unordered) will be denoted by also unordered sequence of it's $n+1$ vertices: $\sigma^{n}=\left(A_{1} \ldots A_{n+1}\right)$. $N_{n}^{(d)}$ is the number of $n$-simplices in $d$-dimensional manifold (may be, infinite). In particular, the 
number of $n$-simplices meeting at given $m$-simplex $\sigma^{m}$ will be denoted as $N_{n}^{(d)}\left(\sigma^{m}\right)$. Now local frames are defined on 4 -simplices $\sigma^{4}=(A B C D E)$. In these frames $a, b, \ldots=0,1,2,3$ are vector indices; metric is $\eta_{a b}=\operatorname{diag}(-1,1,1,1)$ and antisymmetric tensor $\epsilon^{a b c d}$ corresponds to $\epsilon^{0123}=+1 . A \circ B, A * B$ are scalar and dual products of two matrices, respectively; ${ }^{*} B$ is dual matrix:

$$
\begin{gathered}
A \circ B \stackrel{\text { def }}{=} \frac{1}{2} A^{a b} B_{a b} \\
A * B \stackrel{\text { def }}{=} A \circ\left({ }^{*} B\right) \\
{ }^{*} B^{a b} \stackrel{\text { def }}{=} \frac{1}{2} \epsilon^{a b}{ }_{c d} B^{c d}
\end{gathered}
$$

In the local frames the following elements of $S O(3,1)$ are defined: connection matrices $\Omega_{(A B C D)}$ on 3-simplices $(A B C D)$ and curvature matrices $R_{(A B C)}$ on 2-simplices $(A B C)$. Besides, also antisymmetric tensors (bivectors) $v_{(A B C)}$ are defined on 2-simplices: two vectors $l_{1}^{a}, l_{2}^{a}$ form the triangle with bivector

$$
v^{a b}=\epsilon_{a b c d} l_{1}^{c} l_{2}^{d}
$$

whose norm $|v| \stackrel{\text { def }}{=}(v \circ v)^{1 / 2}$ is twice the area of the triangle.

Einstein action for Regge manifold is written in the form [11:

$$
S=\sum_{(A B C)}\left|v_{(A B C)}\right| \arcsin \frac{v_{(A B C)}}{\left|v_{(A B C)}\right|} \circ R_{(A B C)}
$$

Here function arcsin gives angle defect on a triangle in terms of curvature matrix $R$. The latter is product of connection matrices:

$$
R_{(A B C)}=\Omega_{\left(A B C D_{1}\right)}^{\varepsilon_{(A B C) D_{1}}} \ldots \Omega_{\left(A B C D_{r}\right)}^{\varepsilon_{\left(A B C D_{r}\right.}}
$$

where $\varepsilon_{(A B C) D}= \pm 1$ is sign function, whose argument is pair tetrahedron $(A B C D)$ - triangle $(A B C)$. The only requirement imposed on this function is consistency of eqs. of motion for 
$\Omega_{\sigma^{3}}$ which is equivalent for particular $\Omega$ 's to closure condition for 2 -surface of 3 -face $\sigma^{3}$. This condition includes 2 -face bivectors rotated by connection matrices required to transform these bivectors to the same frame. In particular, in the neighbourhood of flat space $\Omega=\mathbf{1}$ it takes the form

$$
\varepsilon_{(A B C) D} v_{(A B C)}+\varepsilon_{(D A B) C} v_{(D A B)}+\varepsilon_{(C D A) B} v_{(C D A)}+\varepsilon_{(B C D) A} v_{(B C D)}=O(\Omega-1) .
$$

Consistency of such the conditions for 3-faces of 4-simplex $(A B C D E)$ sharing common edge $(A B)$ requires that

$$
\varepsilon_{(A B C) D} \varepsilon_{(A B C) E} \varepsilon_{(A B D) E} \varepsilon_{(A B D) C} \varepsilon_{(A B E) C} \varepsilon_{(A B E) D}=-1 .
$$

Next some constraints on bivectors $v$ are required ensuring their tetrad structure. The difficulty is that neighbouring bivectors well may be defined in the different frames; namely, a 4-simplex $\left(A B C D_{0} E_{0}\right)$ exists for each $(A B C)$ where $v_{(A B C)}$ is defined (to reflect this fact let us introduce the more detailed notation

$$
v_{(A B C)} \equiv v_{(A B C) D_{0} E_{0}}
$$

). Therefore it is natural to consider for each $(A B C)$ the set of all $(A B C D E)$ containing this triangle and to define a priori arbitrary corresponding $v_{(A B C) D E}$. Now, what conditions should be fullfilled in order that this set of bivectors would correspond to some Regge manifold where these bivectors are given by (2)? First, consider 4-simplex $(A B C D E)$ and a vertex $A$ in it. The triangles sharing $A$ satisfy relations on dual products of bivectors the same as those for bivectors in the continuum theory at a given point [14]:

$$
\begin{aligned}
& v_{(A B C) D E} * v_{(A B C) D E}=0, \quad \operatorname{perm}(B, C, D, E) \\
& v_{(A B C) D E} * v_{(A B D) C E}=0, \quad \operatorname{perm}(B, C, D, E)
\end{aligned}
$$




$$
\begin{aligned}
& \varepsilon_{(A B C) D} \varepsilon_{(A D E) C} v_{(A B C) D E} * v_{(A D E) B C}+ \\
& \varepsilon_{(A B D) C} \varepsilon_{(A C E) D} v_{(A B D) C E} * v_{(A C E) B D}=0, \quad \operatorname{perm}(B, C, D, E) .
\end{aligned}
$$

Second, the sum of bivectors in any tetrahedron is zero:

$$
\varepsilon_{(A B C) D} v_{(A B C) D E}+\varepsilon_{(D A B) C} v_{(D A B) C E}+\varepsilon_{(C D A) B} v_{(C D A) B E}+\varepsilon_{(B C D) A} v_{(B C D) A E}=0
$$

in any of two 4-simplices $(A B C D E)$ sharing the tetrahedron $(A B C D)$. Not all of the relations (8) - (11) are independent ones since modulo (11) validity of (8) - (10) at any vertex $A$ means their validity at remaining three vertices. If (8) - (11) hold, tensors $v$ in the 4-simplex are bilinears of it's edges just as analogous continuum theory tensors are tetrad bilinears.

Finally, third, we need conditions ensuring unambiguity of linklengths recovered from $v$ in the different 4-simplices. In the continuum theory such the problem did not exist since the tetrad was local function of the bivector. Now we can require continuity of scalar products of bivectors on 3-face $(A B C D)$ shared by 4 -simplices $(A B C D E)$ and $(A B C D F)$ :

$$
\begin{aligned}
& \Delta\left(v_{(A B C) D} \circ v_{(A B C) D}\right) \stackrel{\text { def }}{=} v_{(A B C) D E} \circ v_{(A B C) D E}-v_{(A B C) D F} \circ v_{(A B C) D F}=0, \\
& \operatorname{perm}(A, B, C, D), \\
& \Delta\left(v_{(A B C) D} \circ v_{(A B D) C}\right) \stackrel{\text { def }}{=} v_{(A B C) D E} \circ v_{(A B D) C E}-v_{(A B C) D F} \circ v_{(A B D) C F}=0, \\
& \operatorname{perm}(A, B, C, D) .
\end{aligned}
$$

By (11) there are 6 such independent conditions for each 3-face. This number is sufficient for continuity of it's 6 edges. Eqs. (13), e.g., are sufficient. But the system (13) (modulo (8) - (11)) is still highly reducible: it is sufficient to require continuity of the edges of a triangle on only all but one of tetrahedrons meeting at this triangle to get continuity on all such tetrahedrons.

In terms of only linklengths continuous symmetries of our system are absent since, generally speaking, any change of linklengths means change of geometry. Extension of the set of variables 
by inclusion of connection in our case is compensated by symmetry w.r.t. $S O(3,1)$ rotations of local frames.

Thus, our formulation is characterised by action (3) and by the system of constraints (8) (13) of which we shall below extract irreducible ones.

\section{CONTINUOUS TIME.}

Here we derive the Lagrangian. In fact, it is generalisation on arbitrary Regge manifold of the result of [11] written in bivector notations.

To pass to the continuous time let us divide the set of vertices of Regge manifold into 3dimensional leaves numbered by a parameter $t$ which we call time and tend the step $d t$ between the leaves to zero. The points of the leaf will be denoted by indices $i, k, l, \ldots$ Let us assume the following consistency condition: each 4-dimensional simplex is formed by vertices of only two neighbouring leaves and length of one of it's edges is $O(d t)$. This requires for each vertex $i$ at the leaf $t$ occurence of it's images $i^{+}$in the leaf $t+d t$ and $i^{-}$in the leaf $t-d t$ such that linklengths of $\left(i i^{+}\right)$and $\left(i^{-} i\right)$ are of the order of $d t$. Any such 4-geometry is formed of given 3-leaf as follows. Let us choose any vertex $i$ and consider it's star in 3-leaf, i.e. the set of all the simplices of the leaf containing this vertex. Connect the image $i^{+}$to all the vertices of this star. Then analogous procedure can be repeated with the obtained "mixed" leaf (where vertex $i$ is replaced by $i^{+}$) and with some another vertex $k$. As a result, the leaf arises where two vertices are taken at $t+d t$ and others are at $t$. In analogous way all the rest of vertices can be shifted in time untill we get the leaf all points of which are taken at the time $t+d t$. It is clear that each thus obtained block of 4-geometry filling the space between the leaves $t$ and $t+d t$ is specified by the consequence of the above defined time shifts of vertices. It is remarkable that our Lagrangian will not depend on such the consequence. 
To pass to the limit $d t \rightarrow 0$ let us choose sign function $\varepsilon_{(A B C) D}$ conveniently. In 3dimensional notations put

$$
\varepsilon_{\left(i^{+} k l\right) i}=-1, \quad \varepsilon_{(i k l) i^{+}}=+1
$$

(this unify the form of the kinetic term)

Further, it is convenient when going to the continuous time to assume the continuity condition: if $\left(A_{1} A_{2} \ldots A_{n+1}\right) \rightarrow\left(B_{1} B_{2} \ldots B_{n+1}\right)$ at $d t \rightarrow 0$, then $g_{\left(A_{1} A_{2} \ldots A_{n+1}\right)} \rightarrow g_{\left(B_{1} B_{2} \ldots B_{n+1}\right)}$ for a quantity $g$ defined on $n$-simplices. (Convergence of one simplex to another is understood as convergence of corresponding vertices $A_{j} \rightarrow B_{j}$ and of vectors of links $\left(A_{j} A_{k}\right)$ and $\left.\left(B_{j} B_{k}\right)\right)$.

In particular, let us choose for sign function

$$
\varepsilon_{\left(i^{+} k l\right) m}=\varepsilon_{(i k l) m}, \quad \varepsilon_{(i k l) m^{+}}=\varepsilon_{(i k l) m} .
$$

Also denote

$$
\varepsilon_{i k l} \stackrel{\text { def }}{=} \varepsilon_{\left(i^{+} i k\right) l} .
$$

Then consistency condition for sign function (6) is equivalent to the following one:

$$
\varepsilon_{i k l} \varepsilon_{i k m} \varepsilon_{(i k l) m} \varepsilon_{(i k m) l}=-1 .
$$

Connection on spacelike tetrahedron at $d t \rightarrow 0$ should describe parallel vector transport at infinitesimal distance in time direction and thereby it takes the form

$$
\Omega_{(i k l m)}=\mathbf{1}+f_{(i k l m)} d t .
$$

The same can be written for diagonal tetrahedrons with some vertices shifted to the next time leaf. For continuity reasons corresponding antisymmetric matrices $f$ do not change at such shift (as those describing vector transport at infinitesimally close points and at infinitesimally close directions). But this is even inessential since the resulting Lagrangian turns out to contain 
([11]) only the sum $h_{(i k l m)}$ of $f$ 's over all four types of tetrahedrons - $(i k l m)$ and it's diagonal images with different number of vertices shifted to the next time leaf; for example

$$
h_{(i k l m)}=f_{(i k l m)}+f_{\left(i k l m^{+}\right)}+f_{\left(i k l^{+} m^{+}\right)}+f_{\left(i k^{+} l^{+} m^{+}\right)},
$$

where antisymmetric matrix $h_{(i k l m)}$ is an analog of the continuum GR connection $\omega_{0}$.

The tetrahedron connection is the discrete analog of continuum connection for transport orthogonal to the tetrahedron. Let us denote timelike tetrahedron connection as

$$
\Omega_{i(k l)} \stackrel{\text { def }}{=} \Omega_{(i+i k l)}
$$

(and the same for tetrahedrons differing by time shifts of $k, l$ ).

For bivectors we denote

$$
n_{i k(l m)} d t \stackrel{\text { def }}{=} v_{(i+i k) l m}, \quad \pi_{(i k l) m} \stackrel{\text { def }}{=} v_{(i k l) i^{+} m}
$$

Substituting the limiting form of variables into Regge action we get the Lagrangian where analogs of the terms $\pi \dot{\omega}, h \mathcal{D}_{\alpha} \pi^{\alpha}$ and $n_{\alpha} R^{\alpha}$ of the continuum theory [14 can be viewed denoted below $L_{\dot{\Omega}}, L_{h}$ and $L_{n}$, respectively. Besides, some new terms appear due to the difference of limiting curvature matrices $R$ on spacelike and diagonal triangles from unity巳t. Indeed, write out the finite part of curvature matrix $R_{(i k l)}$ if, e.g., triangle $(i k l)$ is common 2-face of the timelike tetrahedrons $\left(i^{-} i k l\right)$ and $\left(i k^{+} k l\right)$ :

$$
R_{(i k l)}=\Omega_{\left(i^{-} i k l\right)}^{\varepsilon_{(i k l) i^{-}}} \Omega_{\left(i k^{+} k l\right)}^{\varepsilon_{(i k l) k^{+}}}+O(d t)=\Omega_{i(k l)}^{\dagger} \Omega_{k(l i)}+O(d t) .
$$

Normals to the tetrahedrons $\left(i^{-} i k l\right)$ and $\left(i k^{+} k l\right)$ are, generally speaking, different, just as vectors of links $\left(i^{-} i\right)$ and $\left(k k^{+}\right)$are (the latter being analogs of shift-lapse functions at different

\footnotetext{
${ }^{2}$ The closure of these $R$ to unity would be natural to assume for their contribution to $L$ be finite [8]. However, the finiteness can be achieved at noninfinitesimal $R-1$ as well on condition that contributions of neighbouring triangles cancel each other, just as in this work.
} 


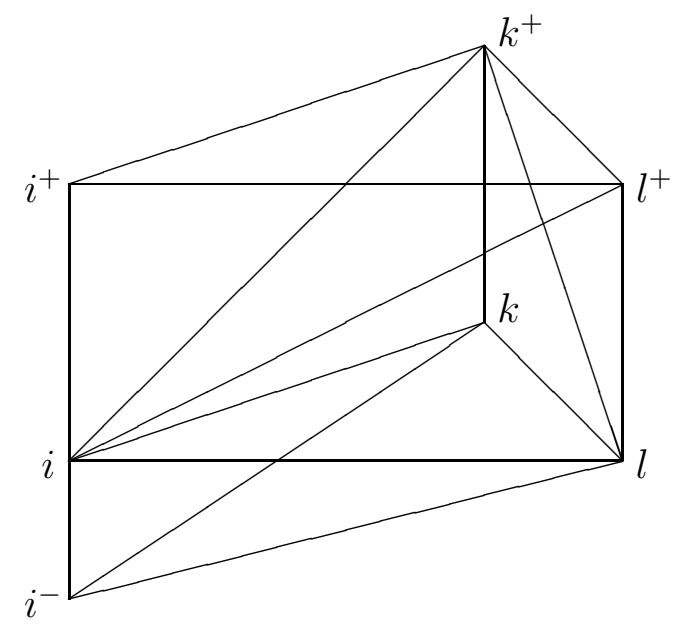

Figure 1: Infinitesimal 3-prism

points), so $\Omega_{i(k l)}$ and $\Omega_{k(l i)}$ do not necessarily coincide. These matrices, however, are not quite independent as follows from the equations of motion for connection; relation between them will also ensure finiteness of the Lagrangian. Indeed, at the infinitesimal variation

$$
\delta \Omega_{k(l i)}=w_{k(l i)} \Omega_{k(l i)} d t, \quad w_{k(l i)}^{\dagger}=-w_{k(l i)}
$$

finite addends to the Lagrangian will arise only from potentially infinite terms (contribution of the triangles with noninfinitesimal area and defects). There are two such terms containing $\Omega_{k(l i)}$ - contributions of $R_{(i k l)}$ and $R_{\left(i k^{+} l\right)}$.

Resulting variation of $L$ is linear in $w_{k(l i)}$ and leads to a constraint on $\Omega, \pi$. Permuting $i, k, l$ we get the system which is solvable [11] and gives

$$
\Omega_{i(k l)}=\Omega_{(i k l)} \exp \left(\phi_{i(k l)} \pi_{(i k l)}+{ }^{*} \phi_{i(k l)}{ }^{*} \pi_{(i k l)}\right),
$$

where $\phi_{i(k l)},{ }^{*} \phi_{i(k l)}$ are parameters. Noninfinitesimal contribution of $(i k l)$ into action (and thus infinite one into $L)$ is proportional to $\phi_{k(l i)}-\phi_{i(k l)}$. Contributions of diagonal triangles differs by cyclic permutations of $i, k, l$ so that the sum vanishes, e.g. (see Fig.1)

$$
\phi_{k(l i)}-\phi_{i(k l)}+\phi_{l(i k)}-\phi_{k(l i)}+\phi_{i(k l)}-\phi_{l(i k)}=0
$$


Important is that these differences are to be multiplied by close up to $O(d t)$ areas of images of $(i k l)$. In the next in $d t$ order to get finite terms in the Lagrangian (below denoted as $L_{\phi}$ ) one should take into account infinitesimal area differences. The latter depend on $n$, the lateral (timelike) 2-face bivectors.

The resulting Lagrangian reads

$$
\begin{aligned}
& L_{\text {Regge }}=L_{\dot{\Omega}}+L_{h}+L_{n}+L_{\phi} \\
& L_{\dot{\Omega}}=\sum_{(i k l)} \pi_{(i k l)} \circ \Omega_{(i k l)}^{\dagger} \dot{\Omega}_{(i k l)} \\
& L_{h}=\sum_{(i k l m)} h_{(i k l m)} \circ \sum_{\text {cycle perm } i k l m} \varepsilon_{(i k l) m} \Omega_{(i k l)}^{\delta_{(i k l) m}} \pi_{(i k l)} \Omega_{(i k l)}^{-\delta_{(i k l) m}} \\
& \left(\delta \stackrel{\text { def }}{=} \frac{1+\varepsilon}{2}\right) \\
& L_{n}=\sum_{i k}\left|n_{i k}\right| \arcsin \frac{n_{i k}}{\left|n_{i k}\right|} \circ R_{i k} \\
& \left(R_{i k}=\Omega_{i\left(k l_{n}\right)}^{\varepsilon_{i k l_{n}}} \ldots \Omega_{i\left(k l_{1}\right)}^{\varepsilon_{i k l_{1}}}, \quad \varepsilon_{i k l_{j}}=\varepsilon_{\left(i k l_{j}\right) l_{j-1}}=-\varepsilon_{\left(i k l_{j}\right) l_{j+1}}\right) \\
& L_{\phi}=-\sum_{(i k l)} \pi_{(i k l) m} \circ \sum_{\operatorname{perm} i k l} \varepsilon_{i k l} \phi_{i(k l)} n_{i k(l m)}
\end{aligned}
$$

(entering last equation scalar products $\pi_{(i k l) m} \circ n_{i k(l m)}$ do not depend on $m$ due to the further considered continuity of scalar products of bivectors). Appearing here in kinetic term bivector $\pi_{(i k l)}$ is $\pi_{(i k l) m}$ at $m=m_{-}(i k l)$, i.e. it is bivector of a triangle $(i k l)$ defined in the one of two tetrahedrons $\left(i k l m_{ \pm}\right)$with the face $(i k l)$ in 3-leaf whose vertices $m_{+}(i k l), m_{-}(i k l)$ (functions of $(i k l))$ are defined according to $\varepsilon_{(i k l) m_{ \pm}}= \pm 1$. We shall also write $\pi_{(i k l) \pm}$ or simply $\pi_{ \pm}$for corresponding bivectors. Bivector $n_{i k}$ is $n_{i k(l m)}$ for some $(l m)$. Thus, $\pi_{-}, \Omega$ are dynamical variables.

For varying in $\phi,{ }^{*} \phi, \Omega$ let us introduce matrices $U=\exp \left(\phi \pi+{ }^{*} \phi{ }^{*} \pi\right)$, so that

$$
\Omega_{i(k l)}=\Omega_{(i k l)} U_{i(k l)}, \quad \phi=\frac{1}{|\pi|} \arcsin \frac{\pi}{|\pi|} \circ U
$$

It is convenient to treat $\Omega, U$ as matrices of general form and take into account the conditions 
of orthogonality and required dependence of $U$ on $\pi$ with the help of Lagrange multipliers by adding to $L_{\text {Regge }}$ the following terms:

$$
\begin{aligned}
L_{\mathrm{rot}}=\sum_{(i k l)} B_{(i k l)} \circ\left(\Omega_{(i k l)}^{\dagger} \Omega_{(i k l)}-\mathbf{1}\right) & +\sum_{i(k l)} P_{i(k l)} \circ\left(U_{i(k l)} \pi_{(i k l)} U_{i(k l)}^{\dagger}-\pi_{(i k l)}\right) \\
& +\sum_{i(k l)} M_{i(k l)} \circ\left(U_{i(k l)}^{\dagger} U_{i(k l)}-\mathbf{1}\right) .
\end{aligned}
$$

Lagrange multipliers are symmetric $(B, M)$ and antisymmetric $(P)$ matrices.

It remains to add to $L_{\text {Regge }}$ with the help of Lagrange multipliers constraints on bivectors (8) - (13) where we shall pass to the notations $\pi, n$ and extract irreducible constraints. Conditions on the dual products $v * v^{\natural}$ where $v^{\natural}$ is $v$ or $v^{\prime}$ result in the constraints $\pi * \pi^{\natural}, \pi * n$ and $n * n^{\natural}$. Since algebraic sum of $\pi$ in the tetrahedron in 3-leaf is zero, there are 6 independent constraints $\pi * \pi^{\natural}$ in the tetrahedron. The number of constraints $\pi * n$ and $n * n^{\natural}$ is 8 and 6 , respectively, in the tetrahedron at each vertex whose shift-lapse vector form given $n$ 's.

The closure condition (11) for 3-leaf tetrahedrons reads

$$
\varepsilon_{(i k l) m} \pi_{(i k l) m}+\varepsilon_{(m i k) l} \pi_{(m i k) l}+\varepsilon_{(l m i) k} \pi_{(l m i) k}+\varepsilon_{(k l m) i} \pi_{(k l m) i}=0
$$

For the timelike tetrahedrons conditions (11) allow us to express variations of bivectors $\pi$ due to time shift of any vertex of 3-leaf in terms of bivectors $n$. These conditions were already used to express variations of $\pi$ appearing when finding $L_{\phi}$.

Subtracting from the number of components of $\pi, n$ (which is $96 N_{3}^{(3)}$ ) the number of constraints (33) and of those of $v * v^{\natural}$ type gives

$$
28 N^{(3)}
$$

for the number of 4-prism parameters. This is natural since any 4-prism is defined by 22 linklengths; in addition, there are 6 rotational degrees of freedom. 


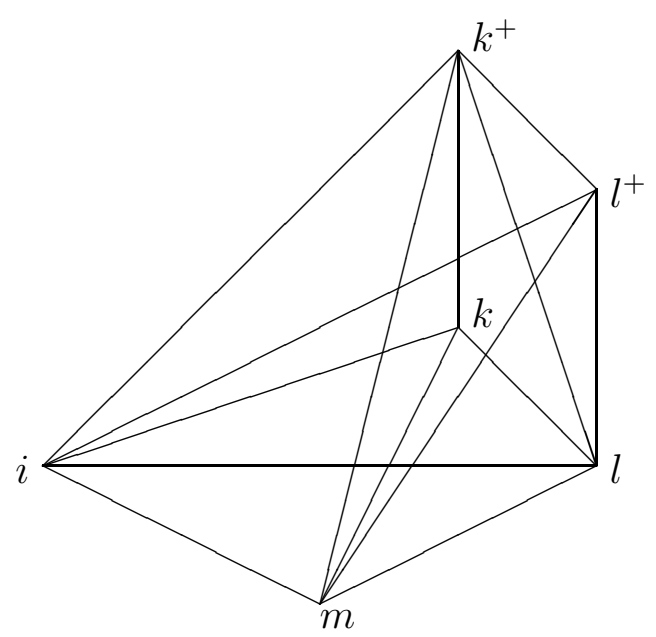

Figure 2: Diagonal 3-face $\left(i k^{+} l m\right)$ - common for 4-simplices $\left(i k k^{+} l m\right)$ and $\left(i k^{+} l l^{+} m\right)$.

Continuity conditions for scalar products (12) and (13) also take different form on spacelike and timelike 3-faces. Namely, continuity on spacelike (and diagonal) faces means constraints with derivatives, whose existence might change dynamical content of theory apart from being simply analog of continuum GR. Fortunately, as it is proved below, given dynamical constraints are consequences of the equations of motion for Lagrangian $L_{\text {Regge }}$ and can be omitted

For example, consider continuity of the values like $v \circ v^{\natural}$ on 3 -face $\left(i k^{+} l m\right)$ (see Fig.2). The difference between bivectors of close spacelike (diagonal) 2-simplices in the same frame on shifting the vertex $k$

$$
\begin{aligned}
D_{k} \pi_{(i k l) m} / d t & \stackrel{\text { def }}{=}\left(v_{\left(i k^{+} l\right) k m}-v_{(i k l) k^{+} m}\right) / d t \\
= & \varepsilon_{k l i} n_{k l(i m)}-\varepsilon_{k i l} n_{k i(l m)},
\end{aligned}
$$

which is an analog of covariant derivative (in fact, already used when finding $L_{\phi}$ ). The difference of bivectors of the same 2-simplices in different frames (an analog of usual derivative)

$$
\delta_{k} \pi_{(i k l) m} \stackrel{\text { def }}{=} v_{\left(i k^{+} l^{+}\right) k m}-v_{(i k l) k^{+} m} .
$$


The continuity conditions connect $\delta \pi$ and $D \pi$ :

$$
\begin{gathered}
\pi_{(i k l) m} \circ\left(\delta_{i} \pi_{(i k l) m}-D_{i} \pi_{(i k l) m}\right)=0, \text { perm } i, k, l ; \\
\pi_{(i l m) k} \circ\left(\delta_{i} \pi_{(i k l) m}-D_{i} \pi_{(i k l) m}\right) \\
+\pi_{(i k l) m} \circ\left(\delta_{i} \pi_{(i l m) k}-D_{i} \pi_{(i l m) k}\right)=0, \text { perm } i, k, l .
\end{gathered}
$$

However, $\delta \pi_{(i k l) m}$ enter equations of motion for $L_{\text {Regge }}$ only in the form of full derivative (and for such $m$ that $\varepsilon_{(i k l) m}=-1$ )

$$
\dot{\pi}_{(i k l)} d t=\left(\delta_{i}+\delta_{k}+\delta_{l}\right) \pi_{(i k l)} .
$$

Therefore constraints (37), (38) are equivalent to relations between $\dot{\pi}$ and $D \pi_{(i k l)}=\left(D_{i}+D_{k}+\right.$ $\left.D_{l}\right) \pi_{(i k l)}$

$$
\begin{aligned}
\pi_{(i k l) m} \circ\left(\dot{\pi}_{(i k l) m}-D \pi_{(i k l)} / d t\right) & =0 \\
\left(D \pi_{(i k l) m} / d t\right. & \left.=\sum_{\text {perm } i k l} \varepsilon_{i k l} n_{i k(l m)}\right), \\
\pi_{(i l m) k} & \circ\left(\dot{\pi}_{(i k l) m}-D \pi_{(i k l) m} / d t\right) \\
+\pi_{(i k l) m} & \circ\left(\dot{\pi}_{(i l m) k}-D \pi_{(i l m) k} / d t\right)=0 .
\end{aligned}
$$

Equations (40), (41) were earlier said to be consequences of the equations of motion for Lagrangian $L_{\text {Regge }}$ supplemented with the rest of constraints on bivectors (without derivatives). Indeed, (40) at $\varepsilon_{(i k l) m}=-1$ arises immediately from $L_{\text {Regge }}$ at the following variation of connection type variables:

$$
\begin{aligned}
\Omega_{(i k l)} & \rightarrow \Omega_{(i k l)} \exp \left(\xi_{(i k l)} \pi_{(i k l)}\right), \\
\phi_{i(k l)} & \rightarrow \phi_{i(k l)}-\xi_{(i k l)}, \quad \text { perm } i, k, l .
\end{aligned}
$$

Namely, the constraint (40) turns out to be added to Lagrangian multiplied by $-\xi_{(i k l)}$. Further, there is area continuity condition $\left|\pi_{(i k l) m_{-}}\right|^{2}=\left|\pi_{(i k l) m_{+}}\right|^{2}$ among the scalar product continuity 
constraints. Differentiating it will lead to (40) also at $\varepsilon_{(i k l) m}=+1$. Besides, in the nondegenerate Regge manifold $N_{2}^{(3)} \geq N_{1}^{(3)}$ (this follows from simple combinatorial discussion with taking into account the fact that each edge is shared by no less then three 2 -faces). Therefore all the links in 3-leaf and, in turn, scalar products of different bivectors $\pi$ can be expressed through triangle areas. Corresponding relations of the type $\pi \circ \pi^{\prime}=f(|\pi|)$ should follow from the below written irreducible set of linear and bilinear constraints on bivectors $\pi, n$ corresponding to Regge manifold. Since we already know how to differentiate areas, using these relations will give derivatives $d\left(\pi \circ \pi^{\prime}\right) / d t$ in terms of $\pi, n$. The obtained relations are purely kinematic ones valid for arbitrary Regge manifold and therefore these are no else than (41).

Thus we have shown that kinematic constraints with derivatives (40), (41) follows from equations of motion for Lagrangian supplemented with constraints without derivatives and should be omitted.

It remains to separate out irreducible conditions of continuity of scalar products (12), (13) on timelike 3-faces. In 3-leaf this corresponds to continuity on (spacelike) triangles. On the triangle $(i k l)$ shared by tetrahedrons $(i k l m),(i k l n)$ we have for the bivectors of timelike and spacelike triangles meeting at vertex $i$ :

$$
\begin{aligned}
\Delta\left(\pi_{(i k l)} \circ \pi_{(i k l)}\right) & \stackrel{\text { def }}{=} \pi_{(i k l) m} \circ \pi_{(i k l) m}-\pi_{(i k l) m} \circ \pi_{(i k l) m}=0, \\
\Delta\left(\pi_{(i k l)} \circ n_{i k}\right) & =0, \\
\Delta\left(\pi_{(i k l)} \circ n_{i l}\right) & =0, \\
\Delta\left(n_{i k} \circ n_{i k}\right) & =0, \\
\Delta\left(n_{i l} \circ n_{i l}\right) & =0, \\
\Delta\left(n_{i k} \circ n_{i l}\right) & =0 .
\end{aligned}
$$

By permutations of $i, k, l$ we get additionally 5 analogous equations at vertices $k, l$ (equation 
(43) remains unchanged). Continuity of edges of tetrahedron $\left(i i^{+} k l\right)$ and, in particular, of the triangle $(i k l)$ follows from (43) - (48). But continuity of the triangle $(i k l)$ follows also from the constraints at vertices $k, l$ as well, that is, some constraints are superfluous. In any case, it is sufficient to keep constraints $\Delta(\pi \circ n)$ at only one vertex of each triangle which gives their full number $2 N_{2}^{(3)}$. The latter is also abundant: the constraint $\Delta\left(\pi_{(i k l)} \circ n_{i k}\right)$ at all others fullfilled expresses continuity of the length of $(i k)$ in 3-leaf which should be stated on all but one triangles $(i k l)$ meeting at this edge. Their full number thus becomes $2 N_{2}^{(3)}-N_{1}^{(3)}$. The constraint $\Delta\left(n_{i k} \circ n_{i l}\right)$ can be associated with length continuity of edge $\left(i i^{+}\right)$. It suffices to impose it on $N_{3}^{(3)}(i)-1$ meeting at $i$ triangles $(i k l)$. Summation over vertices gives $4 N_{3}^{(3)}-N_{0}^{(3)}$ for the number of independent constraints of this type. Finally, constraints $\Delta\left(n_{i k} \circ n_{i k}\right)$ are given on all but one triangles meeting at (ordered) 1-simplex $(i k)$; their full number is $6 N_{2}^{(3)}-2 N_{1}^{(3)}$. The number of constraints $\Delta\left(\pi_{(i k l)} \circ \pi_{(i k l)}\right)$ is $N_{2}^{(3)}$. As a result, the full number of constraints of the type of $\Delta\left(v \circ v^{\natural}\right)$ is $22 N_{3}^{(3)}-3 N_{1}^{(3)}-N_{0}^{(3)}$ (with taking into account that $N_{2}^{(3)}=2 N_{3}^{(3)}$ ). This should be subtracted from (34) to give

$$
6 N_{3}^{(3)}+3 N_{1}^{(3)}+N_{0}^{(3)}
$$

degrees of freedom. Of this number in each 3-leaf $6 N_{3}^{(3)}$ is the number of parameters of local rotations, $N_{0}^{(3)}$ is the number of timelike lengths while $N_{1}^{(3)}$ is the number of spacelike ones; since we consider block of 4-geometry between the two leaves, we take into account here the number of spacelike edges in two leaves, $2 N_{1}^{(3)}$, plus the number of diagonal edges $N_{1}^{(3)}$. However, when we glue different blocks together, we need $N_{1}^{(3)}$ continuity conditions on 3-leaf between them. These are just conditions contained in (40), (41) and shown above to follow from equations of motion. As a result, we have $2 N_{1}^{(3)}+N_{0}^{(3)}$ independent linklengths at arbitrary time as it should be for the Regge 4-manifold constructed of the most general 3-leaf. 
The constraints introduced, $v * v^{\natural}$ and $\sum v, v \circ v^{\natural}$, can be taken into account with the help of Lagrange multipliers by adding to Lagrangian the terms $L_{\text {dual }}$ and $L_{\text {scal }}$, respectively:

$$
\begin{aligned}
L_{\mathrm{dual}} & =\sum_{(i k)(l m)}{ }^{*} \mu_{(i k)(l m)} \pi_{(i k l) m} * \pi_{(i k m) l}+\sum_{i(k l) m}{ }^{*} \nu_{i(k l) m} n_{i k(l m)} * n_{i l(k m)} \\
& +\sum_{i k(l m)}{ }^{*} \nu_{i k(l m)} n_{i k(l m)} * n_{i k(l m)}+\sum_{i k l m}^{*}{ }^{*} \lambda_{i k l m} \pi_{(i k l) m} * n_{i k(l m)} \\
& +\sum_{i k(l m)}{ }^{*} \lambda_{i k(l m)}\left[\varepsilon_{i l k} \varepsilon_{i m k} \pi_{(i l m) k} * n_{i k(l m)}-{ }^{*} \Lambda_{i(k l m)}\right], \\
L_{\text {scal }} & =\sum_{(i k l m)} \eta_{(i k l m)} \circ \sum_{\text {cycle perm } i k l m} \varepsilon_{(i k l) m} \pi_{(i k l) m}+\sum_{(i k l) m} \mu_{(i k l) m}\left(\pi_{(i k l) m} \circ \pi_{(i k l) m}-A_{(i k l)}\right) \\
& +\sum_{i k(l m)} \nu_{i k(l m)}\left(n_{i k(l m)} \circ n_{i k(l m)}-\sigma_{i k}\right)+\sum_{i k l m} \chi_{1}(i k l) \lambda_{i k l m}\left(\pi_{(i k l) m} \circ n_{i k(l m)}-\Lambda_{i k l}\right) \\
& +\sum_{i(k l) m} \chi_{S}(i(k l))\left[\lambda_{i k l m}\left(\pi_{(i k l) m} \circ n_{i k(l m)}-\Lambda_{i k l)}\right)+\lambda_{i l k m}\left(\pi_{(i k l) m} \circ n_{i l(k m)}-\Lambda_{i l k}\right)\right] \\
& +\sum_{i(k l) m} \chi_{\Sigma}(i(k l)) \nu_{i(k l) m}\left(n_{i k(l m)} \circ n_{i l(k m)}-\sigma_{i(k l)}\right) .
\end{aligned}
$$

Here ${ }^{*} \mu, \quad{ }^{*} \nu, \quad{ }^{*} \lambda, \quad \mu, \quad \nu, \quad \lambda, \quad \eta$ and also ${ }^{*} \Lambda, \quad A, \quad \Lambda, \quad \sigma$ are sets of Lagrange multipliers; $\chi_{1}, \quad \chi_{S}, \quad \chi_{\Sigma}$ are characteristic functions of some sets of simplices $S_{1}, \quad S, \quad \Sigma$, arising at constructing irreducible set of constraints above. The $S, \quad \Sigma$ are sets of 2 -simplices with marked vertex, on each of which 2 constraints $\Delta(\pi \circ n)$ and(or $) 1$ constraint $\Delta\left(n \circ n^{\prime}\right)$ are set, respectively. $S_{1}$ is the set of 2 -simplices with marked both vertex and edge on which 1 constraint $\Delta(\pi \circ n)$ is set. It is convenient that the sets $S, \Sigma$ be chosen so that continuity of $\pi_{(i k l)} \circ n_{i k}, \pi_{(i k l)} \circ n_{i l}$ and $n_{i k} \circ n_{i l}$ on necessary number of triangles were fullfilled simultaneously in order that continuity of edges on these triangles would follow immediately. I have check possibility of such choice for two simple examples of 3-leaf: the simplest periodic Regge manifold [16] and simplest closed one - 3-surface of the 4-simplex.

As a result, quasipolinomial Lagrangian of Regge calculus takes the form of the sum of expressions (26), (32), (50) and (51):

$$
L=L_{\text {Regge }}+L_{\text {rot }}+L_{\text {dual }}+L_{\text {scal }}
$$




\section{THE STRUCTURE OF CONSTRAINTS.}

Proceeding to discussion of dynamics, consider full time derivative of some quantity $f$ in the system with Lagrangian (52) which can be written symbolically as

$$
L=\pi \circ \Omega^{\dagger} \dot{\Omega}-H .
$$

Here $H$ is function of $\pi, \Omega$ and other variables. If $f$ is function of $\pi, \Omega$ then it follows with the help of equations of motion that

$$
\frac{d f}{d t}=\{f, H\},
$$

where Poisson brackets for specific form of the kinetic term in $L$ prove to be

$$
\{f, H\}=\pi \circ\left[H_{\pi}, f_{\pi}\right]+H_{\pi} \circ \Omega^{\dagger} f_{\Omega}-f_{\pi} \circ \Omega^{\dagger} H_{\Omega} .
$$

Here indices $\pi, \Omega$ mean corresponding derivative, which over $\pi$ is assumed to be antisymmetrised.

The Hamiltonian $H$, as in the continuum theory, turns out to be linear combination of constraints, i.e. it vanishes on their surface. Indeed, for $L_{\text {dual }}, L_{\text {scal }}, L_{\text {rot }}$ it is so by construction. It is also evident for $L_{h}$, while $L_{n}+L_{\phi}$ is the sum over vertices of the groups of terms $-H_{i}$ each of which is uniform function of degree 1 of the set $n_{i k}$ for all possible $k$ at given $i$. The $n$ 's of this set can be multiplied by some general factor without violating other constraints. This variation leads to Hamiltonian constraint $H_{i}$ :

$$
L_{n}+L_{\phi}=-\sum_{i} H_{i}, \quad H_{i}=0 .
$$

Requiring the constraints be conserved in time allows us to define Lagrange multipliers. Those at II class constraints are defined uniquely and therefore in the absence of matter are zero. Therefore classical dynamics is governed in this case by I class constraints. 
Proceeding to classification of constraints let us first establish continuous symmetries. The latter correspond to occurence of I class constraints. Originally in the full discrete theory we have symmetry w.r.t. $S O(3,1)$ rotations in the local frames in 4 -simplices. In the continuous time limit we have rotations in 4-prisms or, equivalently to say, in their tetrahedron bases; also we have some transformations of $\phi,{ }^{*} \phi$. Tetrahedron rotations $U_{(i k l m)} \in S O(3,1)$ result in

$$
\begin{aligned}
v & \rightarrow U_{(i k l m)} v U_{(i k l m)}^{\dagger}, \\
\Omega_{(i k l)} & \rightarrow\left(U_{(i k l m)} \Omega_{(i k l)}^{\varepsilon_{(i k l) m}} U_{(i k l n)}^{\dagger}\right)^{\varepsilon_{(i k l) m}}, \\
h_{(i k l m)} & \rightarrow U_{(i k l m)} h_{(i k l m)} U_{(i k l m)}^{\dagger}-\dot{U}_{(i k l m)} U_{(i k l m)}^{\dagger},
\end{aligned}
$$

where $v$ is bivector $\pi$ or $n$ in the tetrahedron $(i k l m)$; $(i k l n)$ is another tetrahedron in 3-leaf with the same 2 -face $(i k l)$. It is easy to check that on functions of $\pi, \Omega$ infinitesimal rotations $U=1+u$ are generated by Gaussian constraint $C(u)=-\left.L_{h}\right|_{h \rightarrow u}$ by means of Poisson brackets $\{C(u), \cdot\}($ see $(55))$.

The invariance at shifts $\phi,{ }^{*} \phi$ is due to ambiguity when dividing $\Omega_{i(k l)}$ into symmetric in $i, k, l$ part and rotation $\exp \left(\phi \pi+{ }^{*} \phi^{*} \pi\right)$ not changing $\pi_{(i k l)}$. In particular, symmetry transformations at shift ${ }^{*} \phi$ take the form

$$
\begin{aligned}
{ }^{*} \phi_{i(k l)} & \rightarrow{ }^{*} \phi_{i(k l)}-\zeta_{(i k l)}, \\
\Omega_{(i k l)} & \rightarrow \Omega_{(i k l)} \exp \left(\zeta_{(i k l)}{ }^{*} \pi_{(i k l)}\right), \\
{ }^{*} \mu_{(i k)(l m)} & \rightarrow{ }^{*} \mu_{(i k)(l m)}+\frac{1}{2} \dot{\zeta}_{(i k l)}, \ldots \text { cycle perm } i, k, l \ldots
\end{aligned}
$$

(up to addition full time derivative to the Lagrangian). Generator here is the constraint $\pi_{(i k l)} *$ $\pi_{(i k l)}$, which, although not written explicitly in Lagrangian, is combination of constraints of the type $\pi * \pi^{\prime}$ and $\sum \pi$.

Situation for shift of $\phi$ is complicated by occurence of linear in $\phi$ terms in the Lagrangian: analogous transformations (42) lead, as we have seen, to constraints with generalised velocities 
$\dot{\pi}$. On the other hand, since $N_{2}^{(3)} \geq N_{1}^{(3)}$, there exist $N_{2}^{(3)}-N_{1}^{(3)}$ relations $f_{\alpha}\left(|\pi|^{2}\right)$ on scalar squares of $\pi$. These constraints are consequences of our full set of constraints in $L_{\text {dual }}, L_{\text {scal }}$ and are I class constraints generating transformations (42) for the following particular choice of parameters:

$$
\xi_{(i k l)}=\xi^{\alpha} \frac{\partial f_{\alpha}}{\partial\left(\left|\pi_{(i k l)}\right|^{2}\right)} .
$$

Then, up to the full derivative, the following term in the Lagrangian arises:

$$
\Delta_{\xi} L=\frac{1}{2} \dot{\xi}^{\alpha} f_{\alpha}+\xi^{\alpha} \sum_{(i k l)} \frac{\partial f}{\partial\left(\left|\pi_{(i k l)}\right|^{2}\right)} \pi_{(i k l)} \circ \frac{D \pi_{(i k l)}}{d t}
$$

$(D \pi$ is defined in (40)). First term is here combination of constraints. In the second one the differences of constraints $f_{\alpha}$ between neighbouring 3-leaves arise. These differences are some algebraic constraints on $\pi, n$ and should be combinations from our full set in $L_{\text {dual }}, L_{\text {scal }}$ as well.

Thus, the I class constraints are encountered. These are Gaussian one and kinematic relations for scalar and dual squares of $\pi$. All other constraints, apart from those in $L_{\text {dual }}, L_{\text {scal }}$, should arise when varying $L$ in nondynamical variables $\pi_{+}, n, \quad \phi,{ }^{*} \phi$. Since the latter enter $L$ nonlinearly, the equations obtained do not give, generally speaking, any constraints on dynamical variables $\pi_{-}, \Omega$, but rather simply allow one to express nondynamical variables in terms of dynamical ones. However, an important exception exists: the scale of length of shift-lapse vector at any vertex $i$ enters $L$ linearly. Therefore, first, bivectors $n_{i k}$ at this vertex are defined by given equations only up to the common scale, and, second, variation in this scale gives the above mentioned Hamiltonian constraint (56) at this vertex. This constraint follows by acting on $L$ the following operator:

$$
\sum_{k} n_{i k} \circ \frac{\partial}{\partial n_{i k}}
$$

Substituting into $H_{i}$ the values of nondynamical variables in terms of dynamical ones gives a 
constraint on $\pi_{-}, \Omega$. Nontrivial equations of gravity itself arise in Regge calculus at varying edge lengths, the Hamiltonian constraint corresponding variation in timelike edges. Variation in spacelike and diagonal edges means variation in $\pi_{-}$and gives not the constraints but equations of motion containing time derivatives.

As for the momentum constraints, these might arise, in analogy with continuum GR ( [14), by acting on $L$ the operator

$$
\pi_{(i m k) l} \circ \frac{\partial}{\partial n_{(i k) l m}}-\pi_{(i m l) k} \circ \frac{\partial}{\partial n_{(i l) k m}} .
$$

This operator cancels $L_{\text {dual }}$, but now we have also $L_{\text {scal }}$ not cancelled by this operator. As a result, there are no analogs of the momentum constraints of continuum GR: shift vectors enter $L$ nonlinearly, therefore variation in them allows one only to find these themselves.

Thus our system in the space of dynamical variables $\pi_{-}, \Omega$ is described by $N_{0}^{(3)}$ Hamiltonian constraints $H_{i}, \quad 6 N_{3}^{(3)}$ components of Gaussian constraint $C$ and by additional kinematical constraints on bivectors $\pi_{-}$. The I class constraints are $C, \pi * \pi, f_{\alpha}\left(|\pi|^{2}\right)$. Since $N_{3}^{(3)}=2 N_{2}^{(3)}$, it is convenient to define each $\pi_{-}$in any of two tetrahedrons so that each tetrahedron would contain two bivectors defined in it, $\pi$ and $\pi^{\prime}$. Then other constraints, a priori II class ones, are $H_{i}, \quad N_{3}^{(3)}$ constraints $\pi * \pi^{\prime}$ and $N_{3}^{(3)}$ functions $g_{A}$ expressing scalar products $\pi \circ \pi^{\prime}$ in terms of squares $|\pi|$ :

$$
\pi \circ \pi^{\prime}=g_{A}\left(|\pi|^{2}\right)
$$

It is easy to see that all kinematical constraints mutually commute w.r.t. the brackets (55). Nonzero Poisson brackets arise only between $H_{i}$ 's in different points and between $H_{i}$ and $2 N_{3}^{(3)}$ constraints $\pi * \pi^{\prime}, \pi \circ \pi^{\prime}-g_{A}$. This means that also $2 N_{3}^{(3)}-N_{0}^{(3)}$ I class combinations of functions $\pi * \pi^{\prime}, \quad \pi \circ \pi^{\prime}-g_{A}$ exist. On the whole, there are $6 N_{2}^{(3)}-N_{1}^{(3)}-N_{0}^{(3)}$ I class constraints. As $2 N_{0}^{(3)}$ II class ones we can take, in addition to $H_{i}$, also some $N_{0}^{(3)}$ of products $\pi * \pi^{\prime}$. Without 
taking into account the constraints the number of the degrees of freedom would coincide with the number of canonical pairs $6 N_{2}^{(3)}$. Taking into account the constraints we get this number coinciding with the number of edges $N_{1}^{(3)}$. This should be expectable since, generally speaking, change of the length of any edge means change of geometry of 3-leaf.

We are faced also with some peculiarity connected with that Hamiltonian constraint is II class one. As a result, the length of shift-lapse vector $N$ being Lagrange multiplier at this constraint in empty space is zero. However, in the presence of matter this singularity dissappear. For example, contribution of electromagnetic field $F_{\mu \nu}$ into action containes the terms of the form

$$
g^{00} g^{\alpha \beta} F_{0 \alpha} F_{0 \beta} V
$$

where $V$ is the volume of 4-simplex, $g_{\mu \nu}$ is metric. Since $V \sim N, g^{00} \sim N^{-2}$, the given terms are proportional to $N^{-1}$, so that equations of motion give strictly nonzero value of $N$. One can say that the matter fields prevent the collapse in time axis by developing the pressure from within the 4-simplices.

Vanishing the timelike lengths in empty space leads also in some sence to triviality of classical dynamics in this space. Indeed, in this case Hamiltonian reduces to linear combination of I class constraints. Since all these commute with $\pi \circ \pi$, the areas as well as links do not vary in time. However, normalised bivectors $n /|n|$ have quite complex dynamics. This means that parameters of embedding the 3-leaf into 4-manifold have a nontrivial dynamics.

\section{CONCLUSION.}

Having got Regge calculus in canonical form we can write out puth integral as formal solution to the canonical quantisation problem for this theory. The functional integral measure is defined by volume element in phase space on hypersurface of constraints of the theory and 
contains nonlocal factor which is determinant of the Poisson brackets of II class constraints. The latter are original constraints of theory plus gauge conditions by the number of original I class constraints. One of interesting feature of Regge theory is that (in the case of Euclidean signature) integrations over connections (elements of $\mathrm{SO}(4)$, not of Lee algebra so(4), as in the continuum theory) are finite and one does not need to fix the gauge, that is, to divide by the gauge group volume. In this case the measure factor will be defined by simply the original II class constraints of the theory (Hamiltonian and kinematical ones).

Another, unpleasant feature is that this measure is clearly singular in the vicinity of flat manifold for which symmetry group is larger and classification of constraints changes. Therefore in the vicinity of flat space the perturbation theory does not exist.

\section{ACKNOWLEDGEMENTS.}

The author is grateful to I.B.Khriplovich, V.N.Popov and G.A.Vilkovisky for useful discussions. 


\section{References}

[1] Regge, T.(1961) Nuovo Cimento, 19, 558.

[2] Lund, F., and Regge, T., unpublished.

[3] Collins, P.A., and Williams, R.M.(1973) Phys.Rev., D7, 965.

Collins, P.A., and Williams, R.M.(1974) Phys.Rev., D10, 3537.

[4] Williams, R.M.(1986) Class.Quantum Grav., 3, 853.

[5] Friedman, J.L., and Jack, I.J.(1986) J.Math.Phys., 27, 2973.

[6] Piran, T., and Williams, R.M.(1986) Phys.Rev., 33, 1622.

[7] Porter, J.(1987) Class.Quantum Grav., 4, 375.

Porter, J.(1987) Class.Quantum Grav., 4, 391.

Porter, J.(1987) Class.Quantum Grav., 4, 651.

[8] Brewin, L.(1987) Class.Quantum Grav., 4, 899.

Brewin, L.(1988) Class.Quantum Grav., 5, 839.

[9] Tuckey, P.A., and Williams, R.M.(1988) Class.Quantum Grav., 5, 155.

[10] Tuckey, P.A.(1987) "Independent variables in 3+1 Regge calculus" Cambridge preprint DAMTP 87-32.

[11] Khatsymovsky, V.(1989) Class.Quantum Grav., 6, L249.

Khatsymovsky, V.(1991) Class.Quantum Grav., 8, 1205. 
[12] Bander, M.(1986) Phys.Rev.Lett., 57, 1825.

Bander, M.(1987) Phys.Rev., D36, 2297.

Bander, M.(1988) Phys.Rev., D38, 1056.

[13] Khatsymovsky, V.(1991) "On the two-dimensional model of quantum Regge gravity" Novosibirsk preprint INP 91-40.

Khatsymovsky, V.(1991) "3D Regge gravity as an exactly soluble system" Novosibirsk preprint INP 91-60.

[14] Khatsymovsky, V., "On the polinomial formulations of general relativity", in press.

[15] Sorkin, R.(1975) Journ.Math.Phys., 16, 2432.

Weingarten, D.(1977) Journ.Math.Phys., 18, 165.

[16] Rocek, M., and Williams, R.M.(1981) Phys.Lett., 104B, 31. 